\title{
Utility of liquid biopsy in diagnosing isolated cerebral phaeohyphomycosis: illustrative case
}

\author{
Annie E. Arrighi-Allisan, BA, ${ }^{1}$ Monica M. Vidaurrazaga, MD, ${ }^{2}$ Vincent B. De Chavez, MD, ${ }^{2}$ Clare H. Bryce, MD, ${ }^{3}$ John W. Rutland, BA, ${ }^{1}$ \\ Alberto E. Paniz-Mondolfi, MD, PhD, ${ }^{3}$ Emilia M. Sordillo, MD, PhD, ${ }^{3}$ Michael D. Nowak, MD, ${ }^{3}$ Melissa R. Gitman, MD, MPH, ${ }^{3}$ \\ Risa Fuller, MD, ${ }^{2}$ Emily Baneman, $M D^{2},{ }^{2}$ and Raymund L. Yong, $\mathrm{MD}^{4}$
}

Departments of ${ }^{1}$ Medical Education and ${ }^{3}$ Pathology, Molecular, and Cell-Based Medicine, Icahn School of Medicine at Mount Sinai, New York, New York; and Departments of ${ }^{2}$ Infectious Diseases and ${ }^{4}$ Neurosurgery, Mount Sinai Hospital, New York, New York

\begin{abstract}
BACKGROUND Cladophialophora bantiana is a dematiaceous, saprophytic fungus and a rare but reported cause of intracranial abscesses due to its strong neurotropism. Although it predominantly affects immunocompetent individuals with environmental exposure, more recently, its significance as a highly lethal opportunistic infection in transplant recipients has been recognized. Successful treatment requires timely but often challenging diagnosis, followed by complete surgical excision. Next-generation sequencing of microbial cell-free DNA (cfDNA) from plasma is a novel diagnostic method with the potential to identify invasive fungal infections more rapidly and less invasively than conventional microbiological testing, including brain biopsy.

OBSERVATIONS The authors described the case of a recipient of a liver transplant who presented with seizures and was found to have innumerable ring-enhancing intracranial lesions. The Karius Test, a commercially available method of next-generation sequencing of cfDNA, was used to determine the causative organism. Samples from the patient's plasma identified C. bantiana 6 days before culture results of the surgical specimen, allowing optimization of the empirical antifungal regimen, which led to a reduction in the size of the abscesses.
\end{abstract}

LESSONS The authors' findings suggest that microbial cfDNA sequencing may be particularly impactful in improving the management of brain abscesses in which the differential diagnosis is wide because of immunosuppression.

https://thejns.org/doi/abs/10.3171/CASE21557

KEYWORDS brain abscess; invasive fungal infection; immunosuppression; neurosurgery; next-generation sequencing

Cladophialophora bantiana is a ubiquitous dematiaceous, saprophytic fungus commonly found in soil. ${ }^{1}$ Highly neurotropic, C. bantiana is a rare but recognized cause of intracranial abscess, with just 32 cases reported in North America between 1952 and 2014, mostly in immunocompetent male patients. ${ }^{2,3}$ In immunocompromised patients, particularly recipients of solid organ transplant, $C$. bantiana brain infections have been increasingly reported ${ }^{4,5}$ and may have a mortality rate as high as $80 \%$ because of poor responses to empirical antifungal monotherapy, the presence of multiple abscesses, and delays in establishing the diagnosis, which generally requires brain biopsy. Treatment is largely unsuccessful without complete excision of all abscesses via craniotomy. ${ }^{2}$
Open-ended, next-generation sequencing of microbial cell-free DNA (cfDNA) from plasma has emerged as a novel diagnostic aid in challenging infectious disease scenarios and is now commercially available (Karius Test, Karius Inc.). ${ }^{6,7}$ Compared to a reference standard of all microbial testing results within the initial 7 days of a sepsis alert, microbial cfDNA sequencing boasts a sensitivity and specificity of $92.9 \%$ and $62.7 \%$, respectively, and reduces average time to diagnosis by approximately 40 hours. $^{7}$ There remains, however, a paucity of test performance data in infections manifesting only with brain abscesses, particularly when empirical antimicrobials have already been initiated. ${ }^{8}$ Recent studies have also called into question the ability of microbial cfDNA sequencing to inform clinical management when used in routine

ABBREVIATIONS cfDNA = cell-free DNA; CSF = cerebrospinal fluid; $M P M=$ molecules per microliter; $M R I=$ magnetic resonance imaging INCLUDE WHEN CITING Published January 31, 2022; DOI: 10.3171/CASE21557.

SUBMITTED September 28, 2021. ACCEPTED October 29, 2021.

(C) 2022 The authors, CC BY-NC-ND 4.0 (http://creativecommons.org/licenses/by-nc-nd/4.0/). 
practice, given its lower specificity compared to conventional diagnostics. ${ }^{9,10}$ Here, we describe a case of multiple intracranial $C$. bantiana abscesses in a recipient of a liver transplant in whom Karius testing revealed the diagnosis prior to brain biopsy results and discuss its potential use in rare, invasive fungal infections of the central nervous system.

\section{Illustrative Case}

A middle-aged man presented for medical attention with progressive frontal headaches and new complex seizures. The past medical history was significant for autoimmune hepatitis, primary sclerosing cholangitis, and hepatocellular carcinoma, for which he received an orthotopic cadaveric liver transplant 15 years prior to presentation. His transplant course was complicated by multiple episodes of acute cellular rejection, recurrent autoimmune hepatitis, and, more recently, chronic ductopenic rejection in the setting of intermittent medication noncompliance. At the time of admission, he was on tacrolimus, mycophenolate mofetil, and low-dose prednisone. He possessed no history of travel but did have exposure to mold through his occupation.

On initial examination, he was afebrile, alert, and abulic with a flat affect. He was oriented to person and place but not time. He exhibited word-finding difficulty, an inability to follow complex commands, and bilateral palmar grasp reflexes. Initial laboratory data were significant for normal lymphocyte count, neutrophilia $(84 \%$; normal $40 \%-78 \%$ ), lymphopenia (3\%; normal $15 \%-50 \%$ ), thrombocytopenia $\left(119 \times 10^{3} / \mu \mathrm{L}\right.$; normal $\left.150-450 \times 10^{3} / \mu \mathrm{L}\right)$, and hypoalbuminemia (2.0 g/dL; normal 3.5-4.9 g/dL). Lumbar puncture revealed normal opening pressure and leukocyte count with numerous neutrophils (57\%; normal $<2 \%$ ), elevated protein $(84.6 \mathrm{mg} / \mathrm{dL}$; normal $15-45 \mathrm{mg} / \mathrm{dL}$ ), and normal glucose. Initial magnetic resonance imaging (MRI) revealed innumerable diffusion-restricted lesions with smooth peripheral enhancement, largely localized to the left cerebral hemisphere at the gray-white matter junction (Fig. 1A, left). The patient was started empirically on intravenous trimethoprim-sulfamethoxazole and meropenem on admission.

Further testing was negative for serum cryptococcal, serum coccidioides, and urine Histoplasma antigens. Cerebrospinal fluid (CSF) polymerase chain reaction testing was negative for a large panel of common bacteria and viruses, and cultures showed no growth after 5 days; however, serum and CSF (1,3)-beta-D-glucan (Fungitell test, Associates of Cape Cod Inc.) were found to be elevated (443 and $>500 \mathrm{pg} / \mathrm{mL}$, respectively, reference $<80 \mathrm{pg} / \mathrm{mL}$ ). He was started on liposomal amphotericin B on day 6 but changed to voriconazole because of worsening renal function. On day 9 , repeat brain MRI (Fig. 1A, middle) demonstrated increased size of the abscesses with worsening vasogenic edema, and a Karius Test was performed. Open excisional biopsy of a left temporal abscess was performed the same day. On day 12, while the biopsy results were still pending, Karius assay results returned, detecting C. bantiana at 254 DNA molecules per microliter (MPM), and the patient was immediately started on flucytosine, restarted on liposomal amphotericin $B$, and continued on voriconazole. Three days later, because of worsening renal failure and minimal apparent response, amphotericin was replaced with caspofungin. Histology of the abscess revealed a rim of inflammation with multinucleated giant cells and central necrosis (Fig. 1B, left). Septate and branching fungal hyphae with light brown pigmentation were evident and highlighted by a Fontana Masson stain for melanin (Fig. 1B, right), confirming the presence of a dematiaceous mold. Cultures from the brain biopsy grew C. bantiana on day 18 . Brain MRI on day 22 showed

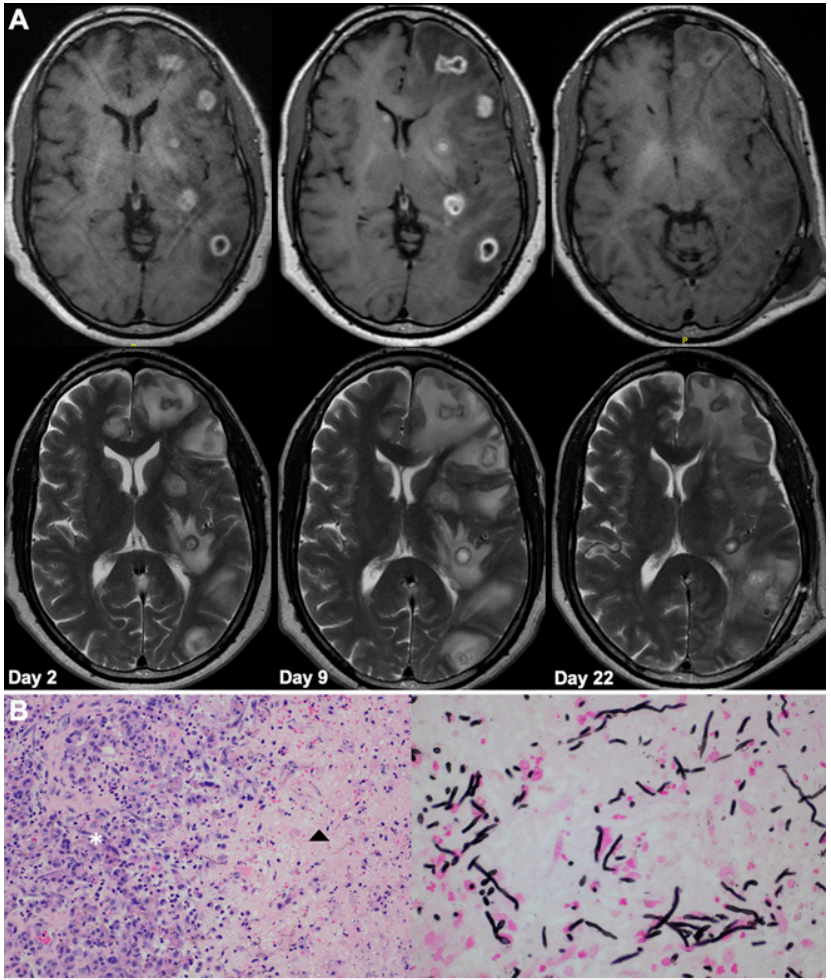

FIG. 1. A: Axial T1-weighted, contrast-enhanced (upper) and T2weighted (lower) MRI performed on days 2, 9, and 22 of admission. B: Left: Inflammation (asterisk) and necrosis (arrowhead) (hematoxylin and eosin stain, original magnification $\times 20$ ). Right: Fungal hyphae (Fontana Masson stain, original magnification $\times 40$ ).

stability of the abscesses and improved vasogenic edema (Fig. 1A, right), but his overall condition deteriorated because of worsening renal failure and thrombocytopenia, causing an acute hemorrhage into the abscess resection cavity on day 23 . He was transitioned to palliative care and expired on day 29.

\section{Discussion}

\section{Observations}

This is the first reported case illustrating the use of plasma microbial cfDNA sequencing to diagnose cerebral abscesses due to C. bantiana, and only the second, to our knowledge, to demonstrate liquid biopsy detection of the causative organism of an isolated intracranial abscess of any etiology. ${ }^{8}$ Because of its rarity and lack of distinguishing clinicoradiographic characteristics, the diagnosis of $C$. bantiana cerebral phaeohyphomycosis takes, on average, 115 days from symptom onset. ${ }^{2}$ Successful treatment is further hampered by the need for an antifungal regimen with both adequate activity against $C$. bantiana and the ability to penetrate the blood-brain barrier. ${ }^{1}$ Complete surgical excision is the only identified independent predictor of survival, ${ }^{2}$ but this aggressive approach differs from that taken in most patients presenting with brain abscesses, in whom stereotactic needle aspiration is generally favored over excision as the initial procedure. ${ }^{11}$ Unfortunately, even when considered, curative surgery proves impossible in approximately one-third of patients because of the presence of multiple or inaccessible lesions. ${ }^{2}$ 
In our case, Karius testing identified C. bantiana 6 days ahead of brain biopsy culture results, prompting us to add flucytosine and liposomal amphotericin $B$ to the empirical voriconazole that was initiated after Fungitell test results were positive 3 days earlier. After 10 days on this regimen, the size of the abscesses stabilized and the worsening left panhemispheric edema reversed, possibly averting an intracranial pressure crisis. The only other reported case of Karius testing identifying the causative organism of an isolated brain abscess occurred in a 49-year-old female recipient of a heart transplant whose single abscess culture indicated Scedosporium apiospermum, another dematiaceous mold. ${ }^{8}$ In contrast to our patient, she had not taken any empirical antifungal therapy at the time of testing. The sensitivity of Karius testing drops significantly with use of antimicrobials within 2 weeks of testing ${ }^{7}$ and with antifungals for Aspergillus. ${ }^{9,12}$ Our case demonstrates that the presence of empirical antifungal agents at the time of testing does not preclude a clinically actionable result, but detection may occur in the range of other asymptomatic organisms ( $<300 \mathrm{MPM})$.

\section{Lessons}

Although we were ultimately unable to provide a surgical cure for our patient, a similar timeline of events in a more typical case of monolesional $C$. bantiana brain abscess could have swayed neurosurgical decision-making away from aspiration or observation and toward early excision, providing a treatment opportunity a conventional workup would have delayed or missed. In a retrospective series of 82 patients, Karius testing had a positive impact on clinical management in six patients. ${ }^{9}$ However, three of these cases involved identification of an invasive fungal organism sooner than standard microbial testing. This report, in conjunction with our case, supports the use of cfDNA to aid in early diagnosis and treatment of infections that are often associated with significant diagnostic delay. Although we found this minimally invasive method of cfDNA detection to be an asset in this patient's course of care, additional larger studies are needed to define the clinical scenarios in which use of the Karius Test will be maximized.

\section{References}

1. Revankar SG, Sutton DA, Rinaldi MG. Primary central nervous system phaeohyphomycosis: a review of 101 cases. Clin Infect Dis. 2004;38(2):206-216.

2. Chakrabarti $A$, Kaur H, Rudramurthy SM, et al. Brain abscess due to Cladophialophora bantiana: a review of 124 cases. Med Mycol. 2016;54(2):111-119.

3. Harrison DK, Moser S, Palmer CA. Central nervous system infections in transplant recipients by Cladophialophora bantiana. South Med J. 2008;101(3):292-296.

4. Ray U, Dutta S, Chakravarty C. A Cladophialophora brain abscess in a renal transplant recipient. Southeast Asian J Trop Med Public Health. 2016;47(5):1026-1031.
5. Gschwend A, Dégot T, Denis J, et al. Brain abscesses caused by Cladophialophora bantiana in a lung transplant patient: a case report and review of the literature. Transpl Infect Dis. 2017;19(6). doi: 10.1111/tid.12787.

6. De Vlaminck I, Martin L, Kertesz M, et al. Noninvasive monitoring of infection and rejection after lung transplantation. Proc Natl Acad Sci U S A. 2015;112(43):13336-13341.

7. Blauwkamp TA, Thair S, Rosen MJ, et al. Analytical and clinical validation of a microbial cell-free DNA sequencing test for infectious disease. Nat Microbiol. 2019;4(4):663-674.

8. Hong DK, Blauwkamp TA, Kertesz M, Bercovici S, Truong C, Banaei N. Liquid biopsy for infectious diseases: sequencing of cellfree plasma to detect pathogen DNA in patients with invasive fungal disease. Diagn Microbiol Infect Dis. 2018;92(3):210-213.

9. Hogan CA, Yang S, Garner OB, et al. Clinical impact of metagenomic next-generation sequencing of plasma cell-free DNA for the diagnosis of infectious diseases: a multicenter retrospective cohort study. Clin Infect Dis. 2020.

10. Wilson MR, Sample HA, Zorn KC, et al. Clinical metagenomic sequencing for diagnosis of meningitis and encephalitis. $N$ Engl J Med. 2019;380(24):2327-2340.

11. Moorthy RK, Rajshekhar V. Management of brain abscess: an overview. Neurosurg Focus. 2008;24(6):E3.

12. Camargo JF, Ahmed AA, Lindner MS, et al. Next-generation sequencing of microbial cell-free DNA for rapid noninvasive diagnosis of infectious diseases in immunocompromised hosts. F1000 Res. 2019;8:1194.

\section{Disclosures}

The authors report no conflict of interest concerning the materials or methods used in this study or the findings specified in this paper.

\section{Author Contributions}

Conception and design: Arrighi-Allisan, Sordillo, Baneman, Yong. Acquisition of data: Arrighi-Allisan, Vidaurrazaga, Bryce, Paniz-Mondolfi, Sordillo, Nowak, Yong. Analysis and interpretation of data: ArrighiAllisan, Vidaurrazaga, De Chavez, Bryce, Paniz-Mondolfi, Sordillo, Gitman, Yong. Drafting the article: Arrighi-Allisan, De Chavez, Bryce, Rutland, Yong. Critically revising the article: Arrighi-Allisan, Vidaurrazaga, De Chavez, Rutland, Paniz-Mondolfi, Sordillo, Fuller, Baneman, Yong. Reviewed submitted version of manuscript: ArrighiAllisan, Vidaurrazaga, De Chavez, Bryce, Rutland, Sordillo, Gitman, Fuller, Baneman, Yong. Approved the final version of the manuscript on behalf of all authors: Arrighi-Allisan. Administrative/technical/material support: Bryce, Nowak. Study supervision: Yong.

\section{Supplemental Information}

Previous Presentations

Portions of this work were presented as a poster at the 88th Annual Meeting of the American Association of Neurological Surgeons, Orlando, FL, August 21-25, 2021.

\section{Correspondence}

Annie E. Arrighi-Allisan: Icahn School of Medicine at Mount Sinai, New York, NY. annie.arrighi-allisan@icahn.mssm.edu. 